\title{
Das Team
}

\section{The Team}

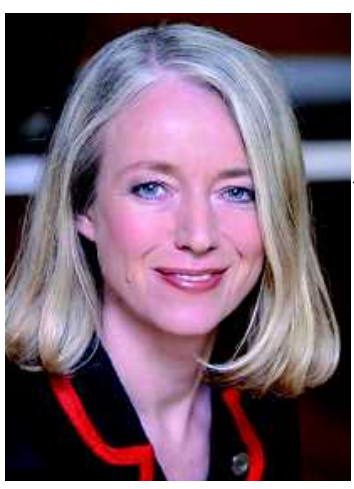

Prof. Dr. Christiane Bayerl
Team-Player! Der neue Terminus, das In-Wort für einen modernen Führungsstil. Bei Einstellungsgesprächen entscheide ich mich für Mitarbeiter, die die Eigenschaft haben im Team erfolgreich zu arbeiten. Die kleinste Einheit eines Teams ist eine Partnerschaft, dann eine Familie bis hin zu einer ganzen Fußballmannschaft. Das Team einer Zeitschrift sind die Autoren, die Herausgeber, der Verlag und vor allem Sie als Leser. Denn nur für Sie schreiben und arbeiten wir im Team. Was Sie lesen, soll für Sie passen, weiterbilden und neugierig machen.

Team-Play heißt nicht, im Team unterzugehen, Gleichmacherei zu betrieben. Vielmehr heißt Team-Play seine Individualität mit allen verrückten und ausgereiften Ideen miteinzubringen. Die Gruppe wird das ihre tun, die Ideen zu werten und zum Reifen zu bringen; und das dient dem gemeinsamen Ziel. Team-Play heißt aber auch sein eigenes Steckenpferd in der Gruppe zu vertreten und das Team damit zu bereichern.

Der Terminus Team-Player beinhaltet, dass es sich um ein „Spiel“ handelt. Spiele machen Spaß und daher trifft die Bezeichnung „Play“. Dennoch, Spiele brauchen Regeln und eine Mannschaft. Mannschaftsspiele sind anstrengend und schweißtreibend. Es gibt Teamsitzungen und $\mathrm{Ar}-$ beitsteilung - also auch Team-Work.

Solche Team-Player hat die Aktuelle Dermatologie schon immer gehabt. Nun hat sich die Mannschaft erweitert und spezialisiert. Wichtige strategische Positionen sind mit Stürmern besetzt, um im Bild einer Fußballmannschaft zu bleiben. Sie werden sie in den nächsten Editorials kennenlernen.

Im April letzten Jahres konnten zwei weitere Klinikdirektoren neu für die Herausgeberschaft der Aktuellen Dermatologie gewonnen werden. Herrn Prof. Dr. med. Christos C. Zouboulis, Chefarzt der Hautklinik und des Immunologischen Zentrums des Städtischen Klinikums Dessau, haben Sie mit Forschungsberichten in der Aktuellen Dermatologie bereits kennengelernt und es sind weitere Manuskripte seiner Mannschaft in Arbeit. Herr Prof. Dr. med. Detlef Zillikens, Direktor der Klinik für Dermatologie und Venerologie des Universitätsklinikums Schleswig-Holstein/Campus Lübeck hat sich ein ehrgeiziges Projekt für die Aktuelle Dermatologie auf die Fahne geschrieben. Er ist in den Startlöchern zur Planung für CME-Manuskripte. Die „süße Last“, Weiterbildungspunkte zu sammeln, ist eine Aufgabe, der wir Dermatologen uns als Niedergelassene, aber auch als Kliniker stellen müssen. Natürlich werden bei Kongressen und regionalen Weiterbildungsabenden Punkte vergeben. Aber das Erreichen der geforderten Gesamtpunkzahl nur durch Reisetätigkeiten ist mit dem ordentlichen Ablauf einer Praxis oder Klinik kaum vereinbar. Also brauchen wir Instrumente, die uns auch bei „Sesshaftigkeit“" Weiterbildung ermöglichen.

Ein heiß umkämpftes aber eigentlich ureigen dermatologisches Arbeitsfeld ist die Histopathologie von Dermatosen und Tumoren der Haut. Eine benachbarte Disziplin versucht sich gerne auch in dieser Kunst. Viele Kliniken haben die Histopathologie in der Dermatologie abgegeben oder abgeben müssen und empfinden das nahezu als schmerzhaft oder als Mangel. Es fehlt die Zusammenschau des klinischen Bildes mit dem Bild am Mikroskop. Daher hat die Stammherausgeberschaft zusätzliche Expertise auch in der Histopathologie und zwar gerade aus dem niedergelassenen Bereich einwerben wollen. Und das ist geglückt in der Person von Herr Dr. med. Volker Mielke, einem passionierten Histopathologen. Er ist tätig in dermatologischer Praxis in Hamburg. So sorgt seine Mitgliedschaft im Herausgebergremium u.a. auch für den Erhalt des Gleichgewichts zwischen „Unileuten“, Klinikdirektoren Städtischer Häuser und niedergelassenen Kollegen in der Herausgeberschaft der Aktuellen Dermatologie.

Nun sind die Spieler alle rekrutiert - es ging auch ohne Ablösesummen, trotz der hohen Qualität der einzelnen Spieler. Damit steht auch die Mannschaftsformation und das Spiel kann beginnen. Es möge für unsere Leser Kenntnisse, Spannung und Freude bringen!

\section{Bibliografie}

DOI $10.1055 / \mathrm{s}-2007-966073$

Akt Dermatol 2007; 33; 1

(c) Georg Thieme Verlag KG .

Stuttgart · New York

ISSN 0340-2541

Korrespondenzadresse

Prof. Dr. med. Christiane BayerI

Klinik für Dermatologie und

Allergologie, HSK-Kliniken

Wilhelm-Fresenius-Klinik

Aukammallee 39

65191 Wiesbaden

Christiane.Bayerl@HSK-Wiesbaden.de 